\title{
DESARROLLO DE LOS VIDEOJUEGOS Y SU INCIDENCIA EN LA ENSEÑANZA DE LOS DEPORTES
}

DEVELOPMENT OF VIDEOGAMES AND THEIR INCIDENCE IN THE TEACHING OF SPORTS

DESENVOLVIMENTO DE VIDEOGAMES E SEU IMPACTO NA EDUCAÇÃO ESPORTIVA

\author{
Edward Alexander Mendoza Ruiz ${ }^{1}$ \\ Oscar Leonardo Acosta Reyes ${ }^{2}$ \\ Jefferson Vargas Aguazaco ${ }^{3}$
}

Resumen

\begin{abstract}
El propósito fundamental de este artículo es presentar una revisión bibliográfica, efectuada en diferentes bases de datos y repositorios a nivel nacional e internacional, que sustente la investigación "La influencia de la consola Nintendo Wii en la enseñanza del drive y revés en tenis de campo en el deporte escolar", llevada a cabo en el Colegio Nuevo San Luis Gonzaga de Bogotá, durante 3 meses, con 12 escolares de 8 a 10 años de edad que no tenían experiencia previa en tenis ni tampoco con video juegos activos relacionados con este deporte, con el fin de conocer si el Wii tiene incidencia positiva o negativa en la enseñanza de estos fundamentos técnicos. Los resultados obtenidos según la prueba International Tenis Number en términos de efectividad mostraron que los videojuegos activos Virtua Tenis 2009 y Wii sport no tuvieron influencia para la enseñanza del golpe de drive. Sin embargo, para el golpe de revés a dos manos hubo una mejoría tanto en efectividad como en construcción global del gesto técnico.
\end{abstract}

Palabras clave: Videojuegos activos; educación; tenis; deporte escolar

\section{Abstract}

The fundamental purpose of this articleis to present a bibliographical review carried out in different databases and repositories at national and international level that support the research "The influence of Nintendo Wii console in teaching drive and reverse field tennis techniques in school sports", carried out at New San Luis Gonzaga School in Bogota for 3 months with 12 school children from 8 to 10 years old, who had no previous experience in tennis nor with active video games in this sport, in order to know if the Wii has positive or negative impact on the teaching of these technical foundation. The results obtained according to the International Tennis Number test in terms of effectiveness showed that the active video games Virtua Tennis 2009 and Wii sport had no influence on the teaching of the drive hit. However, for the two-handed backhand stroke there was an improvement in both effectiveness and overall construction of the technical gesture.

Keywords: active video games; education; tennis; school sport

1 Estudiante de Posgrado del programa Especialización en Pedagogía de la Facultad de Educación de la Universidad Pedagógica Nacional. Docente del Colegio Gimnasio Campestre Los Alpes, Bogotá, Colombia. Correo: edward.mendoza@gimnasiocampestrelosalpes. edu.co. Orcid: 0000-0002-5916-5489.

2 Estudiante de la Licenciatura en Deporte de la Universidad Pedagógica Nacional. Club Deportivo Hanryu Kwan, Bogotá, Colombia. Correo: fef_oacosta088@pedagogica.edu.co. Orcid: 0000-0003-0301-2114.

3 Licenciado en Deporte de la Universidad Pedagógica Nacional. Correo: jvargasa880@pedagogica.edu.co. Orcid: 0000-0003-0343-7390. 


\section{Resumo}

O principal objetivo deste artigo é apresentar uma revisão bibliográfica, realizada em diferentes bancos de dados e repositórios em nível nacional e internacional, que apoie a pesquisa "A influência do console Nintendo Wii no ensino das técnicas drive e backhand no tênis de campo no esporte escolar", realizado no Colégio Nuevo San Luis Gonzaga, em Bogotá, durante 3 meses, com 12 crianças de 8 a 10 anos que não tinham experiência anterior em tênis ou videogame ativo relacionado a esse esporte, para saber se o Wii tem um impacto positivo ou negativo no ensino desses fundamentos técnicos. Os resultados obtidos de acordo com o teste International Tenis Number em termos de efetividade mostraram que os videogames ativos Virtua Tennis 2009 e Wii sport não tiveram influência no ensino do drive. No entanto, para o backhand de duas mãos, houve uma melhora na eficácia e na construção geral do gesto técnico.

Palavras-chave: videogames ativos; educação; tênis; esporte escolar

Para citar este artículo:

Mendoza Ruiz, E.A., Acosta Reyes, O.L. y Vargas Aguazaco, J. (2019). Desarrollo de los videojuegos y su incidencia en la enseñanza de los deportes. Lúdica Pedagógica, 30, 83-92. https://doi.org/10.17227/ludica. num30-11792 


\section{Si puedes mantener la atención de} los niños, puedes educarles.

GLADWELL

\section{INTRODUCCIÓN}

Las investigaciones relacionadas con videojuegos (VJ) de manera global se han centrado en el uso y efecto que estos aparatos tecnológicos tienen a nivel escolar, sociocultural y las cualidades educativas que estos pueden ofrecer (De Souza y Bizelli, 2014; Etxeberría, 1998; Etxeberría, 2009; Gómez, 2007; Marín y García, 2006; Morales, 2009; Salazar y Salazar, 2010; Sánchez, 2008; Pindado, 2005). Al respecto, Del Moral (2010) describe los aportes y potencialidades que presentan determinados vj vinculados al desarrollo de las inteligencias múltiples propuestas por Gardner en el año 2003 (kinestésica, lingüística, musical, espacial, intrapersonal, interpersonal y lógico-matemática). Sin embargo, esta autora también menciona los riesgos que ciertos videojuegos pueden generar en los usuarios como tendencia a la violencia, agresividad, sexismo, racismo, o hacia los pseudoestereotipos desatando en algunos sujetos emociones y comportamientos socialmente no aceptados.

En ese sentido, es bastante polémico y ambivalente pensar el uso de los videojuegos con propósitos educativos, ya que históricamente estos han sido utilizados como mecanismos de simulación de conflictos y formas de producción mercantil, aunque en la actualidad se proyecten también para educar, las opiniones aún siguen divididas. De esta manera, Etxeberría (2009) expresa: "Los primeros pasos de los actuales videojuegos se detectan en los años 40 , cuando los técnicos americanos desarrollaron el primer simulador de vuelo, destinado al entrenamiento de pilotos" (p. 1). De acuerdo con lo anterior, se buscaba acabar los altos costos que implicaba la preparación bélica eliminando así la utilización de grandes cantidades de municiones, equipamientos y recursos que demandan los conflictos, lo que conllevó a través del tiempo a fortalecer la emulación de dichos enfrentamientos produciendo grandes cambios y beneficios en el desarrollo de los mismos.

En la década de los 50 y 60, según Belli y López (2008), los VJ se empezaron a desarrollar principalmente con la finalidad de entretener a las personas.
En algunas universidades de los Estados Unidos, se crean los primeros simuladores de juego por computadora, los cuales a través de su evolución fueron llevados a lugares públicos como bares, salones recreativos, aeropuertos, etc. Posteriormente, con los avances técnicos y tecnológicos, se da inicio en los 70 al uso de los videojuegos en los hogares, dando paso a los juegos domésticos que se podían conectar al televisor. A partir de allí, comienza el desarrollo de una de las industrias más poderosas y lucrativas del ocio y entretenimiento virtual que existe hoy en día.

En un tercer momento, en los inicios del siglo XXI, se destacan las cualidades positivas de los videojuegos para cumplir con propósitos educativos (Casey, 1992; Del Moral, 2010; Díez, 2013; Franco y Nogueira, 2007; Salazar y Salazar, 2010). Este tipo de trabajos reflexionan sobre la conveniencia de utilizar nuevas tecnologías como un medio para la enseñanza en los procesos educativos, específicamente para esta investigación se encuentran evidencias de la re-significación y el sentido otorgado a los VJ como metodologías para la enseñanza de fundamentos técnicos de una disciplina deportiva.

\section{VIDEOJUEGOS Y EDUCACIÓN: RIESGOS Y BENEFICIOS}

En estos momentos se puede considerar que los vJ son uno de los inventos tecnológicos más utilizados por niños, jóvenes y adultos. No obstante, investigaciones como las de Marshall, Biddle, Sallis, McKenzie y Conway, 2002, y Martín, 2007 (citados por Beltrán, Valencia y Molina, 2011) indican que los niños y adolescentes más activos son los que dedican más tiempo a los videojuegos. Un estudio de Del Moral y Villalustre (2008), citado por Del Moral (2010), afirma que "los chicos juegan significativamente más que las chicas con los videojuegos, quienes afirman dedicar algo más de un $20 \%$ de su tiempo libre a esa actividad, mientras que ellas apenas dedican el $8 \% "$ (p. 12). Sustentado en estos datos surgen cuestionamientos como ¿qué tan provechoso o perjudicial puede llegar a ser para la vida de un niño(a) el tiempo que gasta en la interacción con los vJ?

Ahora bien, es pertinente analizar los aspectos negativos que conllevan estos sofisticados aparatos, no solo en contextos escolares, sino en la sociedad y la vida en general. Desde la perspectiva de Beltrán et al. 
(2011), Bustillo (2013), Esnaola (2004) y Etxeberría (1998), se referencia a las consecuencias negativas de los VJ relacionadas con la salud de los jóvenes (enfermedades cardiovasculares, sedentarismo, diabetes tipo II, obesidad, etc.), y la construcción de conductas a partir de una realidad virtual. Por ejemplificar, la violencia se detecta mediante el lenguaje verbal y agresiones en las acciones estratégicas que se pueden extrapolar directa o indirectamente del juego a la vida real.

Respecto al uso excesivo de los vJ, el uso del erotismo como una herramienta de atracción, seducción, promoción y difusión del consumo de videojuegos ha generado múltiples polémicas como la construcción subjetiva de estereotipos físicos (belleza irreal-virtual) por medios audiovisuales que producen trastornos de personalidad alterando la conducta en los niños. Para Bustillo (2013) y Etxeberría (2009), la dedicación desproporcionada de tiempo en el uso de vJ se manifiesta en la necesidad imperiosa de ganar, trayendo como consecuencia el abandonando de forma parcial o total de otras actividades diarias. De la misma forma, los autores consideran que este uso excesivo incita al consumismo, que se evidencia en compras compulsivas de toda clase de productos relacionados con los videojuegos.

Entertainment Software Rating Board (2017), Etxeberría (2009), Iglesias (2011) y Valderrama (2011) plantean clasificaciones o taxonomías para los VJ mostrando los beneficios o perjuicios que estos tienen, sustentados en su finalidad, su aplicación a contextos específicos o su posible carácter educativo. Con todo esto, pareciera paradójica la incorporación de la tecnología en los ambientes escolares para el desarrollo de ciertos contenidos debido a que muchas veces los sistemas educativos manifiestan desacuerdos en la implementación de algunos recursos tecnológicos, particularmente los videojuegos. Existen fuertes posturas a nivel académico y supersticiones culturales que cuestionan la presencia de la tecnología en las aulas y por ende el uso de los vJ en la educación y la sociedad en general.

\section{INVESTIGACIONES SOBRE VJ EN EL CONTEXTO LOCAL, NACIONAL E INTERNACIONAL}

La revisión bibliográfica muestra investigaciones relacionadas con los vj que develan su posible carác- ter formativo en varias áreas del conocimiento, como por ejemplo: el estudio de nuevas herramientas en el aprendizaje musical por medio de los videojuegos (Martínez, 2013), la utilización de un videojuego educativo para la enseñanza de la reproducción humana (Solano, Forero, Guevara y Pinilla, 2013), o el uso de los videojuegos en la enseñanza de la química (Abella y García, 2010).

En el contexto nacional, se observa una ingente producción de estudios relacionados con el uso de los VJ, así se pueden referenciar investigaciones sobre la educación virtual en Colombia (Martínez, Quimbayo y Bustamante, 2010), sobre videojuegos en línea: hacia un estado de la cuestión, desde las ciencias sociales y humanas (Orozco y Patino, 2014), sobre los videojuegos educativos como propuesta de aprendizaje colectivo (Padilla, Collazos, Gutiérrez y Medina, 2012). Se encuentran otros estudios sobre la actividad física en adolescentes y su relación con la agresividad, internet y videojuegos (Chahín y Briñez, 2011), sobre el desarrollo de la creatividad por medio de vJ (Karam, 2010), la construcción de realidades educativas a partir de vJ (Sarmiento, 2012), un estudio clínico sobre los niños y los videojuegos (Orrego, 2007) y un informe sobre producción de vJ en Bogotá (Silva y Chavarro, 2010), entre otras.

En cuanto a los intereses particulares de esta investigación, hay estudios que muestran los aspectos formativos que tienen ciertos vJ en el campo de la educación física y deporte, por ejemplo: Zamora (2000) propone el aprendizaje del reglamento deportivo de atletismo por medio de un videojuego; Corredor (2010) diseña un ambiente virtual para la enseñanza de las normas deportivas del baloncesto y tenis de campo a través de los videojuegos. En un trabajo posterior, Corredor (2013) desarrolla herramientas web en la educación física para implementar ambientes virtuales de aprendizaje en las prácticas motrices. El estudio de Coy, Bautista, Fonseca y Ribeiro (2013) relacionó la influencia de la práctica del tenis mediante el Wii sobre patrones manipulativos en niños de 7 años, esta investigación evidenció que los vj de tenis tienen un impacto significativo en los patrones de atajar y arrojar.

Hoy en día, la implementación de la tecnología es un fenómeno común que ha permeado varios aspectos de la vida, los cuales no están condicionados única- 
mente al consumo, al facilismo de suplir ciertas necesidades, a la diversión, a la economía de esfuerzo, al mejoramiento de la calidad de vida, etc., sino también se debe intentar incorporar con una serie de objetivos y metas claras en los proyectos educativos institucionales de los centros escolares como una posibilidad de contenido del currículo.

\section{LOS VIDEOJUEGOS ACTIVOS}

Los VJ activos, también llamados exergames, usan el movimiento corporal para su desarrollo. Siguiendo a Otero, Nunes, Fonseca, Cavalli y Botelho (2014), "Los VJ activos son consolas que exigen un mayor esfuerzo físico para su jugabilidad en comparación con los videojuegos convencionales" (p. 491). Estos buscan que el usuario tenga un acercamiento interactivo con la consola en pro de que los jugadores dejen de estar sentados por horas frente a la pantalla utilizando solo las manos para mover el joystick (control clásico), su objetivo es incrementar el esfuerzo físico y por ende el gasto calórico superior al de la rutina diaria.

Según Fiallos (2015), "la característica más distintiva de la consola Nintendo Wii es su mando inalámbrico, (Wii mote) el cual puede usarse como un dispositivo que puede detectar movimientos en un plano tridimensional" (p. 14). Los diseñadores del Wii dejan de lado el concepto tradicional de jugar vj revolucionando la forma de interactuar con el usuario, posibilitando otras dinámicas en su uso y funcionamiento. De acuerdo con Garduño A. y Garduño L. (2009), "la realidad virtual generada a través de la pantalla del Wii nos permite reconocer situaciones que afectan a nuestra condición de la realidad física" (p. 10). Es decir, que a través de esta consola se simulan situaciones reales de juego como complemento para la enseñanza de alguna práctica deportiva.

No obstante, los vJ activos han sido poco utilizados para la enseñanza de una habilidad, gesto o técnica deportiva específica, sino que en general se emplean para investigaciones en otras áreas como lo expresan Kliem y Wiemeyer (2010); Middlemas, Basilicato, Prybicien, Savoia, y Biodoglio (2009); Peng, Lin y Crouse (2011); Sohnsmeyer, Gilbrich y Weisser (2010), citados por Santamaría, Salicetti y Moncada (2016), que por medio de la consola Wii se puede mejorar el equilibrio, potencializar la fuerza muscular y asimismo trabajar en procesos de rehabilitación en adultos mayores ya que este aparato tecnológico está relacionado con lo propioceptivo a través de la emulación de movimientos y habilidades para una actividad determinada. Además, los exergames funcionan por medio de las acciones corporales de los usuarios por lo que favorecen a sus practicantes en cuanto a la actividad física el estímulo de variables fisiológicas como el gasto energético, consumo de oxígeno y frecuencia cardiaca. En ese sentido, cabe destacar que la capacidad del Nintendo Wii de simular movimientos puede fundamentar la hipótesis que a través de los exergames se puedan enseñar gestos técnicos específicos de algunas disciplinas deportivas como una alternativa complementaria en los procesos de iniciación deportiva a nivel escolar.

\section{BENEFICIOS DE LOS VIDEOJUEGOS ACTIVOS}

El uso de los vj puede ser considerado como una experiencia interesante en la escuela para la enseñanza de ciertas habilidades, especialmente deportivas. Según Ennis (2013), estos vj "usualmente son motivantes tanto para estudiantes calificados como no cualificados" (p. 152). Un elemento importante y fundamental al referirse a los vJ activos es su capacidad de simulación. De acuerdo con Otero, Longone, Ito, Signori y Silva da Costa (2013), "A través de la realidad virtual de los juegos el educador puede simular prácticas de deportes que no son viables en la escuela" (p. 269). En el mismo sentido, Otero y Silva Da Costa (2010) proponen nuevos ambientes de enseñanza y aprendizaje en la educación física a través del uso de exergames. Liebermann et al. (2010) mencionan ventajas del entrenamiento en entornos virtuales controlados por medio de videojuegos. A su vez, Santos (2015) implementa la utilización de videojuegos en la escuela como recurso didáctico de la educación física. Desde la misma perspectiva, De Oliveira, Gripp y De Lima (2012) manifiestan que los exergames en la educación física pueden desarrollar una cultura digital en la escuela. A su vez, Finco y Fraga (2012) mencionan que se deben romper fronteras en la educación física a través de los videojuegos con interacción corporal. Es así que estos vJ pueden ser una alternativa complementaria que debe estar acompañada de un conocimiento teórico y práctico por parte del profesor para que puedan ser efectivos en el aula. 
INVESTIGACIONES RELACIONADAS CON VIDEOJUEGOS ACTIVOS, LA CONSOLA DE NINTENDO WII Y LA ENSEÑANZA DEL TENIS DE CAMPO A TRAVÉS DE LA TECNOLOGÍA

Un estudio realizado en la Universidad Federal de Pelotas en Brasil determina que hoy en día se están buscando nuevas formas de enseñar a través de la tecnología, una de ellas es el uso de los videojuegos como un medio para la enseñanza del deporte en el contexto escolar (Otero et al., 2014), específicamente Di Tore y Raiola (2012) plantean los exergames como posibilidad en el aprendizaje de habilidades motoras. Fiallos (2015) sugiere el uso de la consola Nintendo Wii en el desarrollo de la motricidad gruesa en niños con problemas congénitos.

Por otra parte, Chen y Hung (2010) analizaron un sistema de captura de movimiento durante el entrenamiento a partir de la enseñanza asistida con el Wii mediante la aceleración de las extremidades para ejercicios de movimientos de entrenamiento de tenis y béisbol. Los resultados evidencian que el sistema reduce la carga de trabajo del entrenador, mejorando la enseñanza y el rendimiento. En la investigación hecha por Li y Liu (2013), citados por Nieblas y Molina (2016), "se analizó el golpeo de drive a partir del video modelado para proporcionar orientación en la enseñanza y formación del tenis, obteniendo efectos positivos" (pp. 116-117). Asimismo, Kim y Roh (2010) exploran las características de los videojuegos interactivos de tenis para personas con discapacidad intelectual, llegando a la conclusión de que estas propuestas aumentan los niveles de habilidad y de motivación, así como la atención y el disfrute.

Nieblas y Molina (2016) han recopilado alrededor de 87 investigaciones científicas en bases de datos reconocidas a nivel internacional (Web of Science y Scopus) asociadas a la implementación de recursos tecnológicos centrados en la enseñanza de las habilidades técnicas del tenis de campo. Al respecto, estos autores mencionan que, "de hecho, la mayoría de los estudios están relacionados con el efecto que tienen las ayudas visuales complementarias para el aprendizaje de las habilidades específicas del tenis" (p. 116). Este tipo de metodologías usadas en los procesos de enseñanza deportiva pueden proporcionar un adecuado asesoramiento para la formación de habilida- des técnicas del tenis, aplicadas al contexto escolar. En ese sentido, es importante reflexionar en que los videojuegos activos como el Nintendo Wii y otros no pueden estar supeditados única y exclusivamente al servicio del entretenimiento de los usuarios, sino que se deben proyectar más allá como un elemento complementario para una educación deportiva.

\section{INICIACIÓN DEPORTIVA EN TENIS DE CAMPO Y SU RELACIÓN CON EL DEPORTE ESCOLAR}

El deporte escolar (DE) es una herramienta valiosa en el momento de establecer un acercamiento a la iniciación y formación deportiva de los sujetos desde edades tempranas, donde la escuela tiene lugar como agente educativo. Por consiguiente, el deporte como uno de los principales medios de desarrollo físico y motriz para niños tiene lugar en diversos ambientes como la escuela o clubes. Para Blázquez et al. (1999), Fraile et al. (2004), Hoyos, Gutiérrez y Pérez (2009), el deporte escolar es una actividad físico/deportiva integradora de carácter formativo realizada por niños/as y jóvenes en edad escolar, dentro y fuera de la escuela, incluso la desarrollada en el ámbito de los clubes o de otras entidades públicas o privadas en sí, en ese sentido, el DE puede conllevar a involucrar a los niños a la iniciación de una práctica deportiva de su interés, la cual puede convertirse con el tiempo en un hábito de vida.

En primera instancia, hay que tener claro qué es lo que sucede de forma general en el desarrollo de los niños, para lo cual Hahn (1988) describe las características de las fases evolutivas en niños y adolescentes. Piaget (2007) explica los estadios del niño desde el nivel sensomotor hasta las operaciones concretas. Además, existen unos periodos sensibles para determinar la edad de iniciación deportiva, donde la escuela desempeña un papel importante para ejecutarlos de la mejor manera posible. Según esto, Blázquez et al. (1999) "plantea que la edad eficaz es a los 9 años y la edad media es a los 11 años” (p. 118). De acuerdo con lo anterior y mencionando a Martens (1986), citado por Blázquez et al. (1999), "para el tenis de campo la edad mínima de los comienzos de la iniciación deportiva seria a los 8 años" (p. 118). Esas cifras de edad pueden variar según las características de la población, la intencionalidad de los objetivos 
planteados y el contexto donde se desarrolle dicha práctica deportiva.

Desde la perspectiva de la Federación Internacional de Tenis ITF (2017), que promueve el "Tenis 10s a través de un programa para jugadores de 10 años y menores" (párr. 1). De acuerdo con ello, este surge como una propuesta didáctica y alternativa para difundir y masificar el tenis de campo. No obstante, Molano (2013) describe que "en el proceso de formación deportiva en tenis de campo existen dos etapas: una de fundamentación y otra de competición, las cuales son complementarias y justifican su razón de ser en los periodos de desarrollo del niño(a)" (p. 80), las cuales se pueden presentar en ámbitos escolares o extraescolares.

Existen trabajos que proponen métodos de enseñanza y aprendizaje alternativos para la iniciación y desarrollo del tenis de campo en el contexto escolar, ya que los contenidos y propósitos de cada etapa en el proceso de formación deportiva son diferentes y presentan distintos componentes de acuerdo con la edad del niño (Julián, Sanz y Del Villar, s. f.; Sanz, Fuentes, Del Villar y Julián, 2004; Sanz y Julián, 2009; Zetou, Koronas, Athanailidis y Koussis, 2012).

Estas investigaciones mencionadas permitieron comprender los múltiples factores que pueden vincular al tenis de campo en las etapas de iniciación y su relación con la escuela, ayudando a entender la importancia de aplicar de manera objetiva dichos elementos teóricos para enriquecer los procesos de enseñanza deportiva.

\section{CONCLUSIONES}

A partir de esta revisión, los videojuegos pueden convertirse en una herramienta tecnológica que estimule y complemente los procesos de enseñanza a nivel deportivo y en otras áreas del currículo. Sin embargo, es fundamental definir su intencionalidad y los procesos de implementación para hacer un uso eficiente de los mismos. El enfoque de esta revisión se proyectó principalmente en el impacto que puede provocar la tecnología en el ámbito educativo, especialmente, para el desarrollo del componente físico y la adquisición de habilidades técnicas en deportistas vinculados a procesos de iniciación. Estos estudios servirán como apoyo para la proyección de nuevas investigaciones relacionadas con la implementación de videojuegos para la enseñanza en el contexto escolar.

Este estudio refleja la necesidad continua de reflexionar sobre nuevas formas de enseñanza, donde la tecnología puede desempeñar un papel activo y complementario para la consecución de dichos objetivos siempre y cuando se tenga clara su intencionalidad educativa. De este modo, cualquier instrumento tecnológico que esté orientado a beneficiar y mejorar aspectos relacionados con la enseñanza deportiva es válido si se utiliza de una forma efectiva para tal fin.

Para concluir, estos referentes teóricos proporcionan otras perspectivas que permiten vislumbrar las posibilidades que podrían presentar ciertos tipos de videojuegos a nivel formativo.

\section{REFERENCIAS}

Abella, L. y García, A. (2010). El uso de videojuegos para la enseñanza de las ciencias, nuevos desafíos al papel docente. Asociación Colombiana para la Investigación en Educación en Ciencias y Tecnología 2.

Belli, S. y López, C. (2008). Breve historia de los videojuegos. Athenea digital, 14, 159-179.

Beltrán, V., Valencia, A. y Molina, J. (2011). Los videojuegos activos y la salud de los jóvenes: revisión de la investigación. Revista Internacional de Medicina, Ciencias de la Actividad Física y el Deporte, 11(41), 1577-0354.

Blázquez, D., Amador, F., Batalla, A., Burriel, J., Carranza, M., Casamort, J., .... Trepat, D. (1999) La iniciación deportiva y el deporte escolar. Barcelona: INDE.

Bustillo, R. (2013). Videojuegos y educación: un reencuentro necesario. [Tesis de grado, Universidad de Cantabria]. Repositorio institucional Unican. http://repositorio.unican.es:8080/xmlui/bitstream/handle/10902/2898/ BustilloCaviaRuben.pdf?sequence $=1 \&$ isAllowed $=\mathrm{y}$

Casey, J. (1992). Using technology and counseling with at-risk youth. ERIC Digests. https://www.ericdigests. org/1992-3/risk.htm

Chahín, N. y Briñez, B. (2011). Actividad física en adolescentes y su relación con agresividad, impulsividad, internet y videojuegos. Psychologia: Avances de la Disciplina, 5(1), 9-23. 
Chen, Y. y Hung, Y. (2010). Using real-time acceleration data for exercise movement training with a decision tree approach. Proceedings of the Eighth International Conference on Machine Learning and Cybernetics, Baoding, 12(15), 3005-3010.

Corredor, S. (2010). Un ambiente virtual para la enseñanza de las normas deportivas. El deporte del Videojuego. http://baloncesto-rosi.blogspot.com.co/

Corredor, S. (2013). Uso de herramientas web en la educación física. Revista Corporeizando, 1(9), 164 - 174.

Coy, L., Bautista, A., Fonseca, L. y Ribeiro, J. (2013). La influencia de la práctica del juego de tenis de realidad virtual sobre los patrones manipulativos en niños de 7 años. Revista Movimiento Científico, 7, 85-92.

Di Tore, P. y Raiola, G. (2012). Exergames and motor skills learning: a brief summary. International Research Journal of Applied and Basic Sciences, 3(6), 1161-1164. http:// www. irjabs.com ISSN 2251-838X

Díez, M. (2013). La accesibilidad en los videojuegos: una asignatura pendiente. Revista Española de Discapacidad, 1(2), 155-158.

Ennis, C. (2013). Implications of exergaming for the physical education curriculum in the 21st century. Journal of Sport and Health Science, 2(3), 152-157.

Entertainment Software Rating Board (2017). Página oficial. http://www.esrb.org/

Esnaola, G. (2004). La construcción de la identidad social a través de los videojuegos: un estudio del aprendizaje en el contexto institucional de la escuela (Tesis doctoral publicada). Universidad de Valencia, España.

Etxeberría, F. (1998). Videojuegos y educación. Reflexiones: Comunicar, 10, 171-180.

Etxeberría, F. (2009). Videojuegos y educación. Revista electrónica. Teoría de la Educación: Educación y Cultura en la Sociedad de la Información, (9), 13-47.

Federación Internacional de Tenis. (2017). Tenis 10s Programa oficial de la ITF. http://www.tennisplayandstay. $\mathrm{com} /$ tennis10s/about-tennis10s/about-tennis10s. aspx

Fiallos, M. (2015). El Nintendo Wii en el desarrollo de la motricidad gruesa en los niños con síndrome de Down de 3 a 4 años. [Tesis de grado, Universidad Técnica de Ambato]. Repositorio institucional UTA. http://repositorio.uta.edu.ec/bitstream/123456789/11429/1/ GABRIELA\%20FIALLOS.pdf
Finco, M. y Fraga, A. (2012). Rompendo fronteiras na Educação Física através dos videogames com interação corporal. Motriz, Rio Claro, 18(3), 533-541.

Fraile, A., Alamo, J., van den Bergh, K., González, J., Graça, A., Kirk, D., .... Theeboom, M. (2004) El deporte escolar en el siglo XXI: análisis y debate desde una perspectiva europea. Barcelona: Biblioteca Tandem, Editorial Grao.

Franco, S. y Nogueira, G. (2007). A nova forma de pensar o jogo, seus valores e suas possibilidades. Revista Pensar a Prática, (2), 323-336.

Garduño, A. y Garduño, L. (2009). La práctica del deporte a través del Wii Nintendo. Revista Electrónica Razón y Palabra, 14(69).

Gómez, M. (2007). Videojuegos y trasmisión de valores. Revista Iberoamericana de Educación, 43(6), 1-10.

Hahn, E. (1988). Entrenamiento con niños. Teoría, práctica, problemas específicos. Barcelona: Ediciones Martínez Roca S. A.

Hoyos, L., Gutiérrez, C. y Pérez, A. (2009). Fundamentación teórica del deporte escolar y desarrollo del deporte escolar en Colombia. España: Alpe.

Iglesias, A. (2011). Desarrollo de videojuegos. Universidad Nacional de Lujan. Buenos aires, Argentina. http://tesis.blanque.com.ar/tesis/Home_files/Tesis_ Alejandro_Adrian_Iglesias.pdf

Julián, J., Sanz, D. y Del Villar, F. (s. f.). La iniciación deportiva al tenis en las sesiones de educación física. In \& EF Innovació en Educació Física. http://www.miguelcrespo.net/temasextra/Tema\%2017.\%20Julian\%20 et $\% 20$ al.\%20Tenis\%20escolar.pdf

Karam, J. (2010). Creatividad y videojuegos: nuevos paradigmas en la investigación de aprendizaje autónomo. Repertorio de Medicina y Cirugía, 20(1).

Kim, J. y Roh, H. (2010). The application of interactive video tennis games on class for the students with intellectual disability. Journal of Adapted Physical Activity and Exercise, 18(2), 1-21.

Liebermann, D., Katz, L., Hughes, M., Bartlett, R., McClements, J. y Franks, I. (2010). Advances in the application of information technology to sport performance. Journal of Sports Sciences, 20(10), 755-769. doi: 10.1080/026404102320675611

Marín, V. y García, M. (2005). Los videojuegos y su capacidad didáctico-formativa. Revista de medios y Educación Pixel-Bit, (26), 113-119. 
Martínez, B., Quimbayo, A. y Bustamante, P. (2010). Educación virtual sentidos, subjetividades y acción pedagógica en contextos multiculticulturales. Mediaciones, 8(10). https://www.researchgate.net/ publication/321103006_Educacion_virtual_sentidos_ subjetividades_y_accion_pedagogica_en_contextos_multiculticulturales/link/5a0d8bf40f7e9b9e33aae8f8/ download

Martínez, C. (2013). Nuevas herramientas en el aprendizaje musical estudio sobre los videojuegos Music on, Donkey konga y Wii music. [Tesis de grado, Universidad Pedagógica Nacional]. Repositorio institucional UPN. http://repository.pedagogica.edu.co/xmlui/bitstream/ handle/123456789/1861/TE-11250.pdf?sequence=1

Molano, P. (2013). Tenis de los pies a la cabeza. Armenia, Colombia: Editorial Kinesis.

Moral del, E. (2010). Aportaciones y riesgos de los videojuegos. Padres y maestros, (331), 12-16. http://revistas.upcomillas.es/index.php/padresymaestros/article/ view/1231

Morales, E. (2009). El uso de los videojuegos como recurso de aprendizaje en educación primaria y teoría de la comunicación. Revista Académica de la Federación Latinoamericana de Facultades de Comunicación Social, 78. https://dialnet.unirioja.es/servlet/ articulo?codigo $=3719704$

Nieblas, J. y Molina, P. (2016). Revisión sistemática de la investigación sobre la enseñanza del tenis. Cuadernos de Psicología del Deporte, 16(2), 111-124.

Oliveira de, A., Gripp, F. y De Lima, M. (2012). Os exergames e a educação física. Escolar na cultura digital. Revista Brasileira de Ciências do Esporte, 34(1), 111-126.

Orozco, R. y Patiño, J. (2014). Investigaciones sobre videojuegos masivos en línea: hacia un estado de la cuestión. Revista Ciencias Humanas, 11, 15-26. http://www.revistas.usb.edu.co/index.php/CienciasHumanas/article/ view/1803/1561

Orrego, J. (2007). Los niños y los videojuegos. Carta de la Salud. Fundación Valle del Lili, 139, 1-4. http:// repository.icesi.edu.co/biblioteca_digital/bitstream/10906/4492/1/139_ni\%c3\%b1os_videojuegos.pdf

Otero, C., Longone, K., Ito, S., Signore, L. y Da Costa, S. (2013). Exergames no currículo da escola: uma metodologia para as aulas de educação Física. XII SBGames. São Pablo. Brasil Outubro. http://www.sbgames.org/ sbgames2013/proceedings/cultura/Culture-7_short.pdf
Otero, C., Nunes, G., Fonseca, B., Cavalli, A. y Botelho, S. (2014). Exergames na Educação Física: ferramentas para o ensino e promoção da saúde. XII SBGames, 491498. http://www.sbgames.org/sbgames2014/files/ papers/culture/full/Cult_Full_Exergames\%20na\%20 Educacao\%20Fisica.pdf

Otero, C. y Silva, S. (2010). Ambientes virtuais de aprendizagem na educação física: uma revisão sobre a utilização de Exergames. Revista Ciências \& Cognição, 15, 76-88. http://pepsic.bvsalud.org/scielo.php?scrip$\mathrm{t}=$ sci_arttext\&pid=S1806-58212010000100008

Padilla, N., Collazos, C., Gutiérrez, F. y Medina, N. (2012). Videojuegos educativos: teorías y propuestas para el aprendizaje en grupo. Revista Ciencia e Ingeniería Neogranadina, 22(1), 139-150.

Piaget, J. (2007). La psicología del Niño. Madrid: Ediciones Morata.

Pindado, J. (2005). Las posibilidades educativas de los videojuegos. Una revisión de los estudios más significativos. Pixel-Bit Revista de Medios y Educación, 26, 55-67. http://www.redalyc.org/pdf/368/36802605.pdf

Salazar, C. y Salazar, F. (2010). Los videojuegos como una herramienta educativa. Habilidad Motriz. COLEF de Andalucía, 35, 41-48.

Sánchez, F. (2008). Videojuegos: una herramienta en el proceso educativo del "Homo Digitalis". Revista Electrónica Teoría de la Educación. Educación y Cultura en La Sociedad de la Información, 9(3).

Santamaría, K., Salicetti, A. y Moncada, J. (2016). Efecto agudo de una práctica "exergame" en el rendimiento del lanzamiento en baloncesto. Retos. Nuevas Tendencias en Educación Física, Deporte y Recreación, (29), 58-60. http://www.redalyc.org/articulo. oa?id=345743464012

Santos, C. (2015). Videogame na escola: a utilização do videogame Xbox e o sensor de movimentos Kinect como recurso didático e estímulo ao processo de aprendizagem nas aulas de educação. Física. Revista Didatica Sistemica. https://periodicos.furg.br/redsis/ article/view/5897

Sanz, D., Fuentes, J., Del Villar, F. y Julián, J. (2004). El tenis en la escuela. Barcelona: Paidotribo.

Sanz, D. y Julián, J. (2009). Metodología de la iniciación al tenis. Propuesta de la RFET para la introducción del tenis en la escuela. Revista Electrónica E-coach, de la Real Federación española de Tenis, 6, 15-22. 
Sarmiento, L. (2012). Videojuegos en línea, construcción de realidades educativas y self. [Tesis de grado, Universidad Nacional de Colombia]. Repositorio institucional UNAL. http://www.humanas.unal.edu.co/red/ files/6913/3780/6723/Videojuegos_en_linea_Realidades_educativas_y_Self_Def_28022012.pdf

Silva y Chavarro (2010). Informe sobre la producción de Juegos de Video en Bogotá. [Tesis de grado, Pontificia Universidad Javeriana]. Repositorio institucional PUJ. https://repository.javeriana.edu. co/bitstream/handle/10554/7521/tesis349. pdf? sequence $=1$ \&isAllowed $=y$

Solano, C., Forero, G., Guevara, J. y Pinilla, R. (2013). Diseño de un videojuego educativo para la enseñanza del proceso de reproducción humana. Revista Vínculos, 10, 342-348. http://revistas.udistrital.edu.co/ojs/index. php/vinculos/article/view/4691/6416
Souza de, C. y Bizelli, J. (2014). A Gamificação do Processo Educativo. Revista GEMInIS, 5(2), 160-176.

Valderrama, J. (2011). Videojuegos y educación: Explorando aprendizajes entre adolescentes. Tesis de maestría. Instituto Tecnológico de Estudios superiores de Occidente, Guadalajara, México. https://rei.iteso.mx/ bitstream/handle/11117/2403/videojuegos-y-educacion.pdf?sequence $=2$

Zamora, C. (2000). Aprendizaje del reglamento deportivo por medio de videojuegos. [Tesis de grado, Universidad Pedagógica Nacional].

Zetou, E., Koronas, V., Athanailidis, I. y Koussis, P. (2012). Learning tennis skill through game Play and Stay in elementary pupils. Journal of Human Sport and Exercise, $7(2)$. 